\title{
Pyruvate dehydrogenase as a therapeutic target for obesity
}

\section{cardiomyopathy}

Andrew JM Lewis MRes ${ }^{1,2}$, Stefan Neubauer FMedSci ${ }^{1}$, Damian J Tyler PhD ${ }^{1,2}$, Oliver J Rider DPhil ${ }^{1,}$ 2 .

${ }^{1}$ Division of Cardiovascular Medicine, Radcliffe Department of Medicine, University of Oxford, OX3 9DU

${ }^{2}$ Department of Physiology, Anatomy and Genetics, Sherrington Road, University of Oxford, OX3 9DU

Corresponding author:

Oliver J Rider

University of Oxford Centre for Clinical Magnetic Resonance Research, John Radcliffe Hospital, Oxford, OX3 9DU, UK

Phone (+44) 1865221869

oliver.rider@gmail.com 


\section{Introduction}

Obesity, defined as a body mass index of $\geq 30 \mathrm{~kg} / \mathrm{m}^{2}$, is associated with a two-fold increased risk of developing heart failure[1] and a spectrum of cardiovascular changes that range from subclinical cardiac impairment to overt ventricular systolic dysfunction[2]. Worldwide, over 2 billion people are either obese or overweight (defined as a body mass index of $25-30 \mathrm{~kg} / \mathrm{m}^{2}$ ), and obesity is thus likely to become a major driver of an increased societal burden of heart failure. No drug has been shown to improve symptoms or outcomes in obesity related heart disease and it therefore represents a major unmet health need.

The term obesity cardiomyopathy is used to describe structural and functional cardiac changes occurring in people with obesity in the absence of other causes. These changes most commonly include ventricular remodelling and impairment of cardiac diastolic function and the obesity epidemic may therefore be an important driver behind the increasing rates of hospital admission for heart failure with preserved ejection fraction (HFPEF), which now outnumber admissions for heart failure with reduced ejection fraction (HFREF)[3]. Obesity can also coexist with, and is likely to exacerbate, heart failure due to other causes (such as ischaemic heart disease or dilated cardiomyopathy, Figure 1) as similar pathophysiological mechanisms apply, though the relationship between obesity and clinical outcomes in advanced heart failure is complex (reviewed in [4]).

Although the pathophysiology of obesity related heart disease is multifactorial[5], an increasing body of evidence suggests that abnormal substrate metabolism may be an important aspect of its development and maintenance and that pharmacological strategies targeting metabolism can improve cardiac structure and function both in experimental models and in small scale human studies. However, there is significant heterogeneity in experimental findings and some important questions remain to be answered prior to definitive human studies. The drugs currently available to manipulate metabolism in obesity have different molecular targets and mechanisms of action, necessitating an understanding of the role of cardiac energy metabolism in the development of obesity cardiomyopathy. 


\section{Cardiac energy metabolism}

In order to fulfil its mechanical function as a pump, the heart generates and consumes chemical energy in the form of adenosine triphosphate (ATP)[6]. The sources of reducing equivalents for ATP synthesis are dietary fuels, including fatty acids and carbohydrates, and, to a lesser extent, amino acids and ketone bodies. In the normal heart in the fasted, resting state, the majority of acetyl CoA entering the tricarboxylic acid cycle is derived from the beta oxidation of free fatty acids (overall 70-90\% of ATP synthesis). Almost all of the remainder is derived from the oxidation of pyruvate arising from glycolysis of carbohydrates, with a small contribution from the glycolysis process itself. In the fed state or during increased workload or hypoxia, there is a significant and adaptive increase in the relative contribution of carbohydrate metabolism to ATP generation.

Although traditionally thought of as a series of individual reactions and pathways, the remarkable flexibility of metabolic networks in the heart is better represented around a limited number of highly interconnected and evolutionarily conserved nodes[7]. The pyruvate dehydrogenase (PDH) enzyme complex is one such critical node, and is an important control point at which the metabolic competition between carbohydrates and fatty acids in the heart is regulated. PDH has a central role in carbohydrate metabolism and cardiac metabolic flexibility, catalysing the oxidative decarboxylation of pyruvate to acetyl CoA and linking glycolysis to the tricarboxylic acid cycle. Obesity diminishes cardiac metabolic flexibility and PDH activity, resulting in an overreliance on fat, which, in addition to increasing the oxygen cost of ATP generation, may contribute to the development of cardiac functional impairment by causing mitochondrial dysfunction, increasing oxidative stress and promoting the deposition of lipids and lipid intermediates. Despite different molecular targets, many pharmacological interventions which indirectly or directly alter PDH flux in obesity are also associated with improved cardiac function, supporting a role for impaired PDH flux in the development of functional impairment in obesity cardiomyopathy[8].

\section{Pyruvate dehydrogenase activity in obesity cardiomyopathy}

The mammalian PDH complex consists of three catalytic subunits, E1-3, and activity is regulated both by allosteric end-product inhibition (by high $\mathrm{NADH} / \mathrm{NAD}^{+}$and acetyl-CoA/CoA ratios) and also by a 
phosphorylation and dephosphorylation cycle. Phosphorylation of PDH occurs at three serine residues on the alpha chain of the E1 subunit of the enzyme complex, and inhibits substrate binding, leading to a decrease in enzyme activity. PDH phosphorylation is catalysed by a family of four pyruvate dehydrogenase kinases (PDKs, designated PDK1-4 in the order in which they were cloned) which are responsive to increased $\mathrm{NADH} / \mathrm{NAD}^{+}$and acetyl $\mathrm{CoA} / \mathrm{CoA}$ ratios, whilst dephosphorylation is catalysed by two pyruvate dehydrogenase phosphatases (PDP1 and PDP2). In the heart, PDK4 is the major regulator of PDH activity[9], and is an important target of peroxisome proliferator-activated receptor (PPAR) signalling pathways. The PPARs belong to the nuclear receptor hormone superfamily and include three members ( $\alpha, \beta / \delta$ and $\gamma)$ which are also differentially expressed according to tissue[10]. PPARa is the dominant isoform in the heart and has a large ligand binding pocket which allows the binding of multiple saturated and unsaturated fatty acid species[11] thus facilitating its function as both an intracellular receptor for lipid molecules and also a transcription factor in the regulation of metabolic gene expression.

In obesity, circulating levels of fatty acids and triacylglycerols are high[12] and, as the heart is a highly efficient scavenger of fatty acids[13], result in increased cardiomyocyte lipid uptake and content[14]. These lipid species activate PPARs, which, in conjunction with heterodimerization with retinoic $\mathrm{X}$ receptors[15] and coactivation[16], lead to transcription of PPAR target genes. PPAR targets include genes involved in every step of cardiac fatty acid metabolism but also include PDK4 (and the remaining PDKs), resulting in a decrease in cardiac PDH activity and metabolic reprogramming of the heart to excessive fat metabolism. PPAR mediated PDK4 transcription is predominantly mediated by the binding of PPAR coactivator/ERR-a to PDK4 promoter regions[17], though obesity is also associated with epigenetic PDK4 promoter modification[18].

Increased PDK levels in the heart are a consistent finding in models of obesity[19, 20], and, in conjunction with low-grade insulin resistance, impaired glucose uptake and allosteric end product inhibition of PDH[21], lead to markedly suppressed cardiac carbohydrate oxidation in animal models[22]; a finding which generally coexists with cardiac systolic and/or diastolic functional impairment. Although the study of cardiac substrate metabolism in in vivo human hearts is challenging, 
positron emission tomography studies are generally consistent with the animal studies and report reductions in cardiac glucose uptake and oxygen efficiency, whilst fat uptake is increased[23, 24]. Importantly, normalization of PDK gene transcript levels[25], increased glucose metabolism, reduced fat oxidation and cardiac functional improvement[26] occur following caloric intake restriction/weight $\operatorname{loss}[27,28]$.

Direct and indirect genetic manipulation of PDH activity has provided further evidence that reduced PDH activity has a role in the development of cardiac functional impairment in animal models of obesity cardiomyopathy. Complete genetic ablation of the heart/skeletal muscle form of PDH in mice leads to embryonic/neonatal mortality[29], which is consistent with the observation that carbohydrate metabolism is the dominant source of reducing equivalents in the developing heart and is essential in the 'fetal phenotype'. Mice with heart and skeletal muscle specific knockout of the alpha-subunit of PDH have no detectable cardiac PDH activity, and, despite embryonic viability, do not survive weaning to standard chow due to heart failure[29]. High fat feeding prolongs the survival of these mice but leads to a severe cardiac phenotype, including ventricular hypertrophy and systolic dysfunction. Furthermore, cardiac specific overexpression of PPAR-a, resulting in markedly elevated PDK4 levels and a significant reduction in glucose oxidation, also causes cardiac dysfunction and hypertrophy[30]. However, cardiac specific overexpression of PDK4, which reduces glucose oxidation and causes cardiac mitochondrial uncoupling, does not cause overt systolic dysfunction which may reflect the development of an adaptive phenotype with a significantly increased capacity for fat oxidation[31]. Although no study has addressed the question of whether genetic approaches to increase PDH activity can improve cardiac diastolic function in obesity, whole body genetic ablation of PDK4 significantly improves glycaemic control[32].

Although PDK mediated inhibition of PDH occurs rapidly following high fat feeding $[33,34]$ and is therefore an early event in obesity, the development of heart failure generally occurs only after a prolonged duration ( 20 years) of obesity[35]. It is therefore likely that chronic PDH inhibition is a component of a 'multiple hit' process leading to the phenotype of obesity cardiomyopathy, with other 
mechanisms including low grade inflammation, neutral and toxic lipid deposition, increased oxidative stress and adverse haemodynamic conditions (Figure 2)[5].

Mechanisms by which reduced PDH activity contributes to functional impairment in obesity cardiomyopathy

\section{Mitochondrial dysfunction}

Cardiac mitochondrial dysfunction is a consistent finding in models of obesity [36] and is an important candidate pathophysiological mechanism of obesity cardiomyopathy. In humans, cardiac energetic status is generally assessed non-invasively using ${ }^{31} \mathrm{P}$ magnetic resonance spectroscopy to measure the concentrations of phosphocreatine (PCr) and ATP with localisation to the myocardium[37]. Obesity is associated with a significant reduction in cardiac PCr/ATP ratio at rest[38, 39], and an even greater reduction during increased cardiac workload[40] when patients with obesity frequently experience undue breathlessness. Thus, although obesity is a state of a chronic excess of circulating metabolic fuel, the heart is paradoxically energy deplete at the level of high energy phosphorus metabolism, a finding which is associated with impaired diastolic function. These observations suggest that obesity causes profound inefficiencies in the pathways linking substrate uptake, oxidation and ATP production.

\section{Increased oxygen cost of fat metabolism}

Although fatty acids have higher potential energy for ATP synthesis than do carbohydrates (298 vs. 223 kcal.mol ${ }^{-1}$ of $\mathrm{C} 2$ units respectively)[41], this comes at a cost of increased oxygen consumption per unit of ATP synthesised. Although stoichiometry would predict that the oxygen cost of ATP generation would be increased by around $12 \%$ for fat oxidation, experimental evidence suggests that the actual cardiac oxygen efficiency is reduced to a much greater degree than this (40-60\% increase in oxygen consumption for a given cardiac workload)[42], a disparity which suggests that additional metabolic mechanisms contribute to oxygen wasting during excessive cardiac fat metabolism.

Impaired oxygen efficiency in obesity cardiomyopathy is of particular importance in the setting of myocardial ischaemia and infarction, and may contribute to the worse clinical outcomes that follow myocardial infarction in obesity[43], despite the tendency for this group to develop myocardial 
infarction at younger age. Although salvage of the central infarct depends primarily on timely reperfusion, the recovery of the peri-infarct tissue is improved by minimising hypoxic exposure[44]. The association between excessive cardiac fat metabolism and increased infarct size has been relatively well detailed in animal models of obesity and/or diabetes[45, 46], as has the beneficial effect of restoring carbohydrate metabolism in these settings[47]. Although human studies using glucose infusions during and following myocardial infarction have yielded conflicting results[48, 49], patients with obesity may be a subgroup who could derive particular benefit from this approach.

\section{Decreased efficiency of oxidative phosphorylation during fat metabolism}

Although potentially limiting during ischemia or during haemodynamic stress, the excess oxygen cost of fat metabolism is unlikely to limit cardiac function in the rested state or during moderate exertion, where diastolic, but generally not systolic, dysfunction is the most common phenotype[40]. Diastolic dysfunction in this setting is likely to be a result of both increased myocardial tissue stiffness due to cardiac lipid accumulation and remodelling, and also from active mechanisms relating to impaired cardiac energetic status and high energy phosphorous metabolism.

Although the majority of ATP produced by the heart is used to support systolic function, diastole is likely to be more susceptible to energetic deficit[50]. The reasons for this lie in the fact that the overall energetic state of the heart is defined not simply by ATP concentration, which is preserved until the development of advanced heart failure. Rather, the heart depends on high rates of both ATP delivery and also of clearance of the products of ATP hydrolysis, ADP and Pi, which inhibit ATPase reactions[51]. The ratio of ATP to ADP and Pi thus determines the available chemical driving force to overcome the thermodynamically unfavourable reactions involved in cardiac contraction and relaxation, including the myosin ATPase and the sarcoplasmic reticulum $\mathrm{Ca}^{2+}$ ATPase (SERCA). Even small increases in ADP concentration due to inhibition of the creatine kinase reaction lead to diastolic dysfunction, likely by slowing myosin ATPase associated cross bridge cycling[52]. In addition, the sarcoplasmic reticulum $\mathrm{Ca}^{2+}$ ATPase also depends upon a high chemical driving force in order to extrude cytosolic calcium and prevent diastolic calcium overload, another important cause of diastolic dysfunction[53]. Thus, although the actin-myosin ATPase consumes a greater quantity of ATP than the 
SERCA, SERCA function requires the highest chemical driving force of all cardiac ATPases (approximately $-53 \mathrm{~kJ} \cdot \mathrm{mol}^{-1}$ )[54] and thus has the earliest susceptibility to energetic deficit.

The provision of a sufficient chemical driving force for ATPase reactions depends not only on the potential energy of the substrate but also on the efficiency of the biochemical processes that link substrate oxidation to mitochondrial redox power. Dominant fat metabolism in obesity is associated with the expression of uncoupling proteins (UCPs) including UCP2, UCP3, adenine nucleotide transporter (ANT) and mitochondrial thioesterase-1 (MTE-1) [55, 56]. These proteins have traditionally been thought to allow proton leak into the mitochondrial matrix, bypassing ATP generating pathways and partially dissipating the proton gradient required for ATP production[57]. In addition to evidence of increased mitochondrial uncoupling in hearts from obese animals, genetic deletion of UCP3 improves mitochondrial efficiency without altering substrate oxidation rates[58]. However, the precise role of UCPs in obesity remain to be fully elucidated, and UCP3 may act as a fatty acid transporter and acetyl CoA buffer[59]. Furthermore mitochondrial uncoupling may have an important protective role in mitigating oxidative stress in obesity, as, when electron supply and the mitochondrial membrane potential exceed ADP availability or demand for ATP synthesis[60], excess electrons escape by reducing molecular oxygen, leading to the production of hydrogen peroxide. A further potential role for UCP3 in the prevention of cardiac arrhythmias under conditions of excessive lipid availability was recently described[61]. Additional mechanisms linking dominant fat metabolism to inefficient oxidative phosphorylation include the inefficient delivery of reducing equivalents to complex I by betaoxidation[62] and oxidative post translational modifications of complex II[63], leading to impaired function.

Despite the profound metabolic consequences of dominant fat metabolism, the healthy heart is energetically resilient and it is therefore unlikely that an acute shift in substrate metabolism from carbohydrates to fat is sufficient in isolation to cause energetic deficit leading to functional deficit[64]. However, the presence of additional, subclinical myocardial pathology in obesity might significantly increase the energetic cost of contraction and relaxation, and, as a shift towards predominant carbohydrate metabolism (reversion to the so-called "fetal phenotype") is an early, and likely adaptive, 
metabolic shift in hypertrophied and mildly failing hearts, the presence of insulin resistance and inhibition of the machinery of carbohydrate metabolism may abrogate this shift, exacerbating energetic deficit.

\section{Lipid uptake and accumulation}

Due to the limited capacity of the heart to store fatty acids, continuous energy generation depends on efficient extraction of fatty acids from a continuous circulatory supply. Fatty acid uptake into cardiomyocytes occurs either via diffusion across the cell wall or by fatty acid specific transporters, including fatty acid translocase (FAT/CD36) and fatty acid transport protein 1 . Once in the intracellular space, fatty acids are activated by esterification to form acyl CoA, and the subsequent conversion to long chain acyl carnitine and transport into the mitochondria is regulated by carnitine palmitoyltransferase 1 (CPT1), which is located on the outer mitochondrial membrane. CPT1 activity, and hence fatty acid oxidation in the heart, is inhibited by malonyl CoA which is in turn regulated by acetyl CoA carboxylase, which catalyses the synthesis of malonyl CoA from acetyl CoA. Although malonyl CoA is a potent inhibitor of CPT1 in vitro, it is less active in vivo and may compete with fatty acyl CoA[65]. When myocardial uptake of fatty acids exceeds capacity for oxidation, the excess fatty acids increase the intracellular fatty acyl CoA pool, which is diverted into non-oxidative processes including ceramide and diacylglycerol synthesis[8], which may independently act as deleterious signalling and proapoptotic molecules. Animal models of obesity demonstrate increased fatty acid transporter expression, cardiac lipid accumulation and impaired contractile function[14, 66]. Furthermore, genetic overexpression of cardiac fatty acid transport protein 1 is sufficient to cause increased fatty acid uptake and contractile dysfunction in the absence of systemic metabolic perturbation[67]. Ablation of FAT/CD36 is protective against high fat diet induced cardiac lipotoxicity[68], whilst genetic overexpression of adipose triglyceride lipase, leading to a reduction in fatty acid uptake and oxidation, conferred protection against high fat feeding induced lipotoxicity and contractile dysfunction[69]. In human studies, obesity was associated with increased myocardial triglyceride content measured using proton magnetic resonance spectroscopy, and the myocardial triglyceride content was in turn associated with impaired diastolic function[70]. These data and others 
suggest that cardiac lipid accumulation in obesity contributes to the development of functional impairment, possibly by directly increasing myocardial stiffness and/or by inducing deleterious signalling programmes. Importantly, stimulation of cardiac PDH flux may cause a reciprocal reduction in CPT1 activity, and hence mitochondrial fatty acid uptake and oxidation, by increasing acetyl CoA and therefore malonyl CoA[71], though the effects of this approach upon cardiac lipid accumulation and function remain to be formally tested.

\section{Pharmacological strategies to alter PDH activity in obesity}

Inhibitors of pyruvate dehydrogenase kinases

Dichloroacetate is a pyruvate mimetic which inhibits PDK activity and is the drug most commonly used to increase PDH activity in experimental settings. Although the mechanism by which DCA inhibits PDK activity varies between PDK isoforms, DCA binds to the pyruvate domain of PDK4 with ADP, and inhibits activity by locking the active site in a closed conformational state[72]. In vivo, DCA causes rapid inhibition of PDK activity, with measurable activity within 30 minutes of oral or parenteral administration[73]. In addition to its rapid effects on PDH via PDK inhibition, DCA may also have a further mechanism of action by reducing PDC turnover[74], though the mechanisms responsible for this effect remain to be elucidated. DCA has established antihyperglycaemic effects in diabetes[75, 76], increases PDH flux and improves diastolic function in rodent models of diabetes[77]. Although DCA has whole body effects, thus altering the circulating substrate profile, isolated perfused heart studies also show that DCA improves cardiac function, supporting a direct mechanism of action[78]. Furthermore, an acute infusion of DCA improved cardiac oxygen efficiency and mechanical function in humans with heart failure[79].

The potential for clinical translation of dichloroacetate for obesity cardiomyopathy is limited by a narrow therapeutic window and toxicity, and some trials of this drug in mitochondrial diseases have been terminated early due to significant peripheral neuropathy[80]. Glutathione transferase zeta (GSTz1)/maleylacetoacetate isomerase (MAAI) has recently been identified as an important regulator of dichloroacetate breakdown, and identification of GSTz1/ MAAI haplotype status might enable more individualised dosing, reducing the risk of toxicity[81]. 
Sodium phenylbutyrate is a novel PDK inhibitor, and improved PDH activity in a model of congenital lactic acidosis[82]. Sodium phenylbutyrate is already approved for human use in some jurisdictions, but may not be appropriate for cardiac applications as it is primarily active against PDK1-3 with lower activity against PDK4[83], although the effect of phenylbutyrate on cardiac PDH activity has not been specifically tested. Recent developments in the understanding of the structure of PDKs and renewed interest in PDK inhibition as a therapeutic strategy in oncology are likely to lead to the development of new small molecule PDK inhibitors, with important potential roles in cardiac and metabolic science[84].

\section{Fatty acid oxidation inhibitors}

Inhibitors of fatty acid oxidation indirectly increase cardiac carbohydrate metabolism and PDH activity, and are used as anti-anginal agents in some countries. Inhibition of fatty acid oxidation leads to a decrease in beta oxidation, thus decreasing acetyl CoA and activating PDH leading to an improvement in cardiac oxygen efficiency. Perhexiline and Etomoxir inhibit fatty acid oxidation by binding to CPT1 and 2 (competitively and non- competitively respectively), whilst Trimetazidine is a weaker inhibitor of CPT-1 and inhibits fatty acid oxidation primarily by reducing the activity of specific enzymes of beta oxidation[85]. The effects of inhibiting fatty acid oxidation upon cardiac metabolism and function have most commonly been studied using Trimetazidine, which causes substantial reductions in fatty acid oxidation with reciprocal increases in glucose oxidation[85]. Consistent with its effects upon metabolic substrate metabolism, Trimetazidine improves cardiac energetics during experimental low flow ischaemia[86] and in human heart failure[87]. The use of Trimetazidine to improve cardiac substrate and energetic metabolism in obesity has been limited by concern that inhibition of fatty acid oxidation in the presence of excess fatty acid levels might cause accumulation of toxic intermediate of lipid metabolism, including ceramides and diacylgylercols. These species, in addition to their proapoptotic effects, might exacerbate insulin resistance thus abrogating metabolic benefit. However, recent studies of Trimetazidine did not support these concerns and demonstrated instead that this drug prevented the development of both cardiac hypertrophy and diastolic dysfunction in a murine model of obesity cardiomyopathy[88]. Furthermore, Trimetazidine had no effect upon skeletal muscle toxic lipid 
intermediates or whole body insulin resistance[89], whilst Trimetazidine prevented palmitate induced ceramide accumulation and mitochondrial dysfunction and improved ATP production in cultured cardiomyocytes[90]. These findings suggest that Trimetazidine may be an attractive drug for clinical studies in obesity cardiomyopathy, with lesser effects upon skeletal muscle metabolism.

Ranolazine, an inhibitor of the cardiac late sodium current, also has inhibitory effects on fatty acid oxidation leading to an indirect increase in cardiac glucose utilisation, which might contribute to the antianginal properties of this drug[91]. Ranolazine has not been tested in obesity cardiomyopathy, but improved exercise capacity and some indices of diastolic function in diastolic heart failure[92].

\section{Glucagon like peptide 1 (GLP-1) analogues}

The incretin mimetics are an emerging class of antihyperglycaemic drugs for diabetes, which stimulate the glucagon like receptor 1 receptor (GLP-1R). The antihyperglycaemic effect of stimulation of the GLP-1R in diabetes is mediated by an increase in glucose dependent insulin section, by activation of G-protein coupled receptors in islet beta cells[93]. However, GLP-1Rs are expressed widely in peripheral tissues, including the heart, and the cardiovascular effects of incretin analogues are of increasing interest. In isolated rat hearts, recombinant GLP-1 increases glucose uptake and enhances recovery following ischaemia[94], whilst, in a canine model of heart failure, recombinant GLP-1 increases cardiac glucose uptake, insulin sensitivity and contractility[95]. In a rodent model of obesity cardiomyopathy, the GLP-1 analogue Liraglutide increased cardiac PDH activity, normalised energetic reserve and improved diastolic function[96].

In addition to administering GLP-1 analogues, the bioavailability of endogenous GLP-1 can also be enhanced by inhibiting dipeptidylpeptidase-4 (DPP-4), which catalyses GLP-1 breakdown. The DPP-4 inhibitor Linagliptin improved diastolic function in a rodent obesity model[97], whilst a different DPP4 inhibitor, MK0626, also improved diastolic function in obese mice[98].

In addition to their effects on myocardial substrate metabolism, GLP-1 analogues activate protective cell signalling pathways, ameliorating inflammation, NF- $\mathrm{kB}$ translocation and other markers of apoptosis, fibrosis and hypertrophy[99]. One further benefit of GLP-1 analogues is to promote satiety, 
thus reducing energy intake and leading to weight loss[100]. These data suggest that GLP-1 analogues may be an effective therapeutic approach for obesity cardiomyopathy, though the relative contribution of their multiple putative mechanisms of action remain to be fully elucidated.

\section{Conclusions}

Obesity cardiomyopathy poses a major public health problem and lacks therapeutic options. Although the pathophysiology of obesity is complex and undoubtedly multifactorial, a growing body of evidence suggests that pharmacological and non-pharmacological strategies that directly or indirectly normalise cardiac substrate metabolism by increasing PDH flux are associated with improvement in cardiac energetics and function, though uncertainties remain with respect to the reciprocal effects on fat metabolism, lipid accumulation and other parameters. A more complete understanding of the metabolic profile of lipid and carbohydrate metabolism in human obesity cardiomyopathy from new metabolic imaging technologies is likely to facilitate the identification of patient groups most likely to benefit from these therapies.

\section{Expert opinion}

Although pharmacological metabolic therapy for cardiovascular disease has been a focus of significant interest for many years, no drug is yet routinely used clinically to improve cardiac function/cardiovascular outcomes. The understanding of cardiac energy metabolism has improved dramatically in recent decades, and the importance of metabolic signalling in adaptive and maladaptive responses to environmental stimuli as well as ATP generation is now appreciated. Obesity cardiomyopathy has particular promise for metabolic therapy, as alterations in cardiac substrate metabolism towards excessive fatty acid oxidation at the expense of glucose oxidation via PDH appear to be an important causal factor in the development of decreased myocardial efficiency and cardiac functional impairment. This understanding has in large part been driven by reductionist approaches using animal models with and without genetic modification, though there remains significant heterogeneity between the findings from animal and human studies - for example, many mouse models of obesity develop overt systolic dysfunction which is less frequently seen in obese humans in the absence of alternate causes. There is also significant variability in the occurrence of other 
pathophysiological features of obesity cardiomyopathy including insulin resistance and lipid accumulation between different models, underscoring the complexity of metabolic heart disease and the need for detailed phenotyping and comparison between species.

The successful translation of therapies targeting PDH activity to human obesity cardiomyopathy will require a deeper understanding of the interplay between cardiac substrate metabolism, lipid deposition, energy generation and cardiac function in obese humans as well as continued animal and cell based studies. Established imaging technologies, including PET tracer uptake studies, ${ }^{1} \mathrm{H}$ spectroscopy for myocardial triglyceride content and ${ }^{31} \mathrm{P}$ spectroscopy for ATP metabolism, have provided insight into the metabolic phenotype of human obesity cardiomyopathy, and may become useful for assessing response in phase II and dose ranging studies in obesity cardiomyopathy. Novel imaging technologies, including hyperpolarized $\left[1-{ }^{13} \mathrm{C}\right]$ pyruvate spectroscopy which can directly measure cardiac PDH activity in vivo, are also likely to contribute significantly to mechanistic understanding and the identification of optimal dosing strategies and subgroups of patients most likely to derive benefit. However, although these studies will be helpful in identifying the most promising drugs and doses for phase III studies, the reported paradoxical improvement in survival in obese humans with heart failure highlights that definitive trials will need to demonstrate an improvement in cardiovascular outcomes, rather than simply improvement in cardiac metabolism or function, to justify widespread use.

Of the drugs currently available to manipulate PDH activity, fatty acid oxidation inhibitors, particularly Trimetazidine, and incretin mimetics, particularly Liraglutide, have the greatest preclinical evidence of benefit. Both classes of drug have effects beyond increasing PDH activity however, and the relative importance of these effects remains to be understood. The pleiotropic effects of Liraglutide, including the promotion of satiety and weight loss may be a significant additional benefit. The currently available PDK inactivators have limitations that might preclude widespread human use, though new small molecule inhibitors are likely to become available and may have a more favourable side effect profile.

In conclusion, obesity cardiomyopathy poses a significant healthcare burden and lacks pharmacological therapeutic options, though restoration of PDH activity is associated with favourable effects upon cardiac metabolism and function in animal models, supporting a potential role as a therapeutic target. 
The convergence of a deeper understanding of cardiac energy metabolism from preclinical models and novel metabolic imaging technologies to enable deep human cardiac metabolic phenotyping bode well for the application of drugs targeting PDH activity in obesity cardiomyopathy. 


\section{References}

1. Kenchaiah, S., et al., Obesity and the risk of heart failure. New England Journal of Medicine, 2002. 347(5): p. 305-313.

2. Alpert, M.A., Obesity Cardiomyopathy:: Pathophysiology and Evolution of the Clinical Syndrome. The American journal of the medical sciences, 2001. 321(4): p. 225-236.

3. Owan, T.E., et al., Trends in prevalence and outcome of heart failure with preserved ejection fraction. New England Journal of Medicine, 2006. 355(3): p. 251-259.

4. Rayner, J., S. Neubauer, and O. Rider, The paradox of obesity cardiomyopathy and the potential for weight loss as a therapy. Obesity Reviews, 2015.

5. Abel, E.D., S.E. Litwin, and G. Sweeney, Cardiac remodeling in obesity. Physiological reviews, 2008. 88(2): p. 389-419.

6. Neubauer, S., The failing heart — an engine out of fuel. New England Journal of Medicine, 2007. 356(11): p. 1140-1151.

7. Caetano-Anollés, G., H.S. Kim, and J.E. Mittenthal, The origin of modern metabolic networks inferred from phylogenomic analysis of protein architecture. Proceedings of the National Academy of Sciences, 2007. 104(22): p. 9358-9363.

8. WU, P., et al., Starvation and diabetes increase the amount of pyruvate dehydrogenase kinase isoenzyme 4 in rat heart. Biochem. J, 1998. 329: p. 197-201.

9. Barger, P.M. and D.P. Kelly, PPAR signaling in the control of cardiac energy metabolism. Trends in cardiovascular medicine, 2000. 10(6): p. 238-245.

10. Varga, T., Z. Czimmerer, and L. Nagy, PPARs are a unique set of fatty acid regulated transcription factors controlling both lipid metabolism and inflammation. Biochimica et Biophysica Acta (BBA)-Molecular Basis of Disease, 2011. 1812(8): p. 1007-1022.

11. Jensen, M.D., et al., Influence of body fat distribution on free fatty acid metabolism in obesity. Journal of Clinical Investigation, 1989. 83(4): p. 1168.

12. Vyska, K., et al., Regional myocardial free fatty acid extraction in normal and ischemic myocardium. Circulation, 1988. 78(5): p. 1218-1233. 
13. Zhou, Y.-T., et al., Lipotoxic heart disease in obese rats: implications for human obesity. Proceedings of the National Academy of Sciences, 2000. 97(4): p. 1784-1789.

14. Keller, H.R., et al., Fatty acids and retinoids control lipid metabolism through activation of peroxisome proliferator-activated receptor-retinoid X receptor heterodimers. Proceedings of the National Academy of Sciences, 1993. 90(6): p. 2160-2164.

15. Huss, J.M., et al., Estrogen-related receptor $\alpha$ directs peroxisome proliferator-activated receptor $\alpha$ signaling in the transcriptional control of energy metabolism in cardiac and skeletal muscle. Molecular and cellular biology, 2004. 24(20): p. 9079-9091.

16. Wende, A.R., et al., PGC-1 $\alpha$ coactivates PDK4 gene expression via the orphan nuclear receptor ERRQ: a mechanism for transcriptional control of muscle glucose metabolism. Molecular and cellular biology, 2005. 25(24): p. 10684-10694.

17. Kirchner, H., et al., Altered promoter methylation of PDK4, IL1 B, IL6, and TNF after Rouxen Y gastric bypass. Surgery for Obesity and Related Diseases, 2014. 10(4): p. 671-678.

18. Young, M.E., et al., Impaired long-chain fatty acid oxidation and contractile dysfunction in the obese Zucker rat heart. Diabetes, 2002. 51(8): p. 2587-2595.

19. Buchanan, J., et al., Reduced cardiac efficiency and altered substrate metabolism precedes the onset of hyperglycemia and contractile dysfunction in two mouse models of insulin resistance and obesity. Endocrinology, 2005. 146(12): p. 5341-5349.

20. Kolter, T., I. Uphues, and J. Eckel, Molecular analysis of insulin resistance in isolated ventricular cardiomyocytes of obese Zucker rats. American Journal of PhysiologyEndocrinology And Metabolism, 1997. 273(1): p. E59-E67.

21. Chatham, J.C. and A.-M.L. Seymour, Cardiac carbohydrate metabolism in Zucker diabetic fatty rats. Cardiovascular research, 2002. 55(1): p. 104-112.

22. Peterson, L.R., et al., Effect of obesity and insulin resistance on myocardial substrate metabolism and efficiency in young women. Circulation, 2004. 109(18): p. 2191-2196.

23. Labbé, S.M., et al., Increased myocardial uptake of dietary fatty acids linked to cardiac dysfunction in glucose-intolerant humans. Diabetes, 2012. 61(11): p. 2701-2710. 
24. Rosa, G., et al., Reduced PDK4 expression associates with increased insulin sensitivity in postobese patients. Obesity research, 2003. 11(2): p. 176-182.

25. Viljanen, A.P., et al., Effect of caloric restriction on myocardial fatty acid uptake, left ventricular mass, and cardiac work in obese adults. The American journal of cardiology, 2009. 103(12): p. 1721-1726.

26. Fenk, S., et al., Successful weight reduction improves left ventricular diastolic function and physical performance in severe obesity. International heart journal, 2015(0).

27. Sankaralingam, S., et al., Lowering body weight in obese mice with diastolic heart failure improves cardiac insulin sensitivity and function: implications for the obesity paradox. Diabetes, 2015. 64(5): p. 1643-1657.

28. Sidhu, S., et al., Tissue-specific pyruvate dehydrogenase complex deficiency causes cardiac hypertrophy and sudden death of weaned male mice. American Journal of Physiology-Heart and Circulatory Physiology, 2008. 295(3): p. H946-H952.

29. Finck, B.N., et al., The cardiac phenotype induced by PPAR $\alpha$ overexpression mimics that caused by diabetes mellitus. The Journal of clinical investigation, 2002. 109(109 (1)): p. 121130.

30. Chambers, K.T., et al., Chronic inhibition of pyruvate dehydrogenase in heart triggers an adaptive metabolic response. Journal of Biological Chemistry, 2011. 286(13): p. 1115511162.

31. Jeoung, N.H. and R.A. Harris, Pyruvate dehydrogenase kinase-4 deficiency lowers blood glucose and improves glucose tolerance in diet-induced obese mice. American Journal of Physiology-Endocrinology and Metabolism, 2008. 295(1): p. E46-E54.

32. Crewe, C., M. Kinter, and L.I. Szweda, Rapid inhibition of pyruvate dehydrogenase: an initiating event in high dietary fat-induced loss of metabolic flexibility in the heart. PloS one, 2013. 8(10): p. e77280.

33. Crewe, C.L., M. Kinter, and L. Szweda, Adaptation of the Heart to High-Dietary Fat: Rapid Inhibition Of Pyruvate Dehydrogenase. The FASEB Journal, 2013. 27(1_MeetingAbstracts): p. 1192.24 . 
34. Scaglione, R., et al., Left ventricular diastolic and systolic function in normotensive obese subjects: influence of degree and duration of obesity. European heart journal, 1992. 13(6): p. 738-742.

35. Lopaschuk, G.D., C.D. Folmes, and W.C. Stanley, Cardiac energy metabolism in obesity. Circulation research, 2007. 101(4): p. 335-347.

36. Ritov, V.B., et al., Deficiency of subsarcolemmal mitochondria in obesity and type 2 diabetes. Diabetes, 2005. 54(1): p. 8-14.

37. Neubauer, S., et al., $31 P$ magnetic resonance spectroscopy in dilated cardiomyopathy and coronary artery disease. Altered cardiac high-energy phosphate metabolism in heart failure. Circulation, 1992. 86(6): p. 1810-1818.

38. Perseghin, G., et al., Abnormal left ventricular energy metabolism in obese men with preserved systolic and diastolic functions is associated with insulin resistance. Diabetes Care, 2007. 30(6): p. 1520-1526.

39. Rider, O., et al., Effects of weight loss on myocardial energetics and diastolic function in obesity. The international journal of cardiovascular imaging, 2013. 29(5): p. 1043-1050.

40. Rider, O.J., et al., Effects of catecholamine stress on diastolic function and myocardial energetics in obesity. Circulation, 2012. 125(12): p. 1511-1519.

41. Ashrafian, H., J.D. Horowitz, and M.P. Frenneaux, Perhexiline. Cardiovascular drug reviews, 2007. 25(1): p. 76-97.

42. Mjøs, O.D., J.K. Kjekshus, and J. Lekven, Importance of free fatty acids as a determinant of myocardial oxygen consumption and myocardial ischemic injury during norepinephrine infusion in dogs. Journal of Clinical Investigation, 1974. 53(5): p. 1290.

43. Das, S.R., et al., Impact of body weight and extreme obesity on the presentation, treatment, and in-hospital outcomes of 50,149 patients with ST-segment elevation myocardial infarction: results from the NCDR (National Cardiovascular Data Registry). Journal of the American College of Cardiology, 2011. 58(25): p. 2642-2650. 
44. McVEIGH, J.J. and G.D. Lopaschuk, Dichloroacetate stimulation of glucose oxidation improves recovery of ischemic rat hearts. American Journal of Physiology-Heart and Circulatory Physiology, 1990. 259(4): p. H1079-H1085.

45. Thakker, G.D., et al., Effects of diet-induced obesity on inflammation and remodeling after myocardial infarction. American Journal of Physiology-Heart and Circulatory Physiology, 2006. 291(5): p. H2504-H2514.

46. Greer, J.J., D.P. Ware, and D.J. Lefer, Myocardial infarction and heart failure in the $d b / d b$ diabetic mouse. American Journal of Physiology-Heart and Circulatory Physiology, 2006. 290(1): p. H146-H153.

47. Maarman, G., et al., Effect of chronic CPT-1 inhibition on myocardial ischemia-reperfusion injury (I/R) in a model of diet-induced obesity. Cardiovascular drugs and therapy, 2012. 26(3): p. 205-216.

48. Malmberg, K., et al., Randomized trial of insulin-glucose infusion followed by subcutaneous insulin treatment in diabetic patients with acute myocardial infarction (DIGAMI study): effects on mortality at 1 year. Journal of the American College of Cardiology, 1995. 26(1): p. $57-65$.

49. Selker, H.P., et al., One-year outcomes of out-of-hospital administration of intravenous glucose, insulin, and potassium (GIK) in patients with suspected acute coronary syndromes (from the IMMEDIATE [Immediate Myocardial Metabolic Enhancement During Initial Assessment and Treatment in Emergency Care] Trial). The American journal of cardiology, 2014. 113(10): p. 1599-1605.

50. Phan, T.T. and M. Frenneaux, The pathophysiology of diastolic heart failure. F1000 biology reports, 2010. 2.

51. Ingwall, J.S. and R.G. Weiss, Is the failing heart energy starved? On using chemical energy to support cardiac function. Circulation research, 2004. 95(2): p. 135-145.

52. Tian, R., et al., Failure to maintain a low ADP concentration impairs diastolic function in hypertrophied rat hearts. Circulation, 1997. 96(4): p. 1313-1319. 
53. Bers, D.M., Sarcoplasmic reticulum Ca uptake, content and release, in Excitation-contraction coupling and cardiac contractile force. 2001, Springer. p. 161-202.

54. Kammermeier, H., High energy phosphate of the myocardium: concentration versus free energy change, in Cardiac Energetics. 1987, Springer. p. 31-36.

55. Murray, A.J., et al., Dietary long-chain, but not medium-chain, triglycerides impair exercise performance and uncouple cardiac mitochondria in rats. Nutrition \& metabolism, 2011. 8(1): p. $1-9$.

56. Boudina, S., et al., Mitochondrial Energetics in the Heart in Obesity-Related Diabetes Direct Evidence for Increased Uncoupled Respiration and Activation of Uncoupling Proteins. Diabetes, 2007. 56(10): p. 2457-2466.

57. Murray, A.J., et al., Uncoupling proteins in human heart. The Lancet, 2004. 364(9447): p. 1786-1788.

58. Boudina, S., et al., UCP3 Regulates Cardiac Efficiency and Mitochondrial Coupling in High Fat-Fed Mice but Not in Leptin-Deficient Mice. Diabetes, 2012. 61(12): p. 3260-3269.

59. Essop, M.F., et al., Hypoxia-induced decrease of UCP3 gene expression in rat heart parallels metabolic gene switching but fails to affect mitochondrial respiratory coupling. Biochemical and biophysical research communications, 2004. 314(2): p. 561-564.

60. Muoio, D.M., Metabolic inflexibility: when mitochondrial indecision leads to metabolic gridlock. Cell, 2014. 159(6): p. 1253-1262.

61. Nabben, M., et al., Lack of UCP3 does not affect skeletal muscle mitochondrial function under lipid-challenged conditions, but leads to sudden cardiac death. Basic research in cardiology, 2014. 109(6): p. 1-12.

62. Veech, R.L., The therapeutic implications of ketone bodies: the effects of ketone bodies in pathological conditions: ketosis, ketogenic diet, redox states, insulin resistance, and mitochondrial metabolism. Prostaglandins, leukotrienes and essential fatty acids, 2004. 70(3): p. 309-319. 
63. Sverdlov, A.L., et al., High fat, high sucrose diet causes cardiac mitochondrial dysfunction due in part to oxidative post-translational modification of mitochondrial complex II. Journal of molecular and cellular cardiology, 2015. 78: p. 165-173.

64. Mjøs, O.D., Effect of free fatty acids on myocardial function and oxygen consumption in intact dogs. Journal of Clinical Investigation, 1971. 50(7): p. 1386.

65. Carley, A.N., H. Taegtmeyer, and E.D. Lewandowski, Matrix Revisited Mechanisms Linking Energy Substrate Metabolism to the Function of the Heart. Circulation research, 2014. 114(4): p. 717-729.

66. Christoffersen, C., et al., Cardiac lipid accumulation associated with diastolic dysfunction in obese mice. Endocrinology, 2003. 144(8): p. 3483-3490.

67. Chiu, H.-C., et al., Transgenic expression of fatty acid transport protein 1 in the heart causes lipotoxic cardiomyopathy. Circulation research, 2005. 96(2): p. 225-233.

68. Yang, J., et al., CD36 deficiency rescues lipotoxic cardiomyopathy. Circulation research, 2007. 100(8): p. 1208-1217.

69. Pulinilkunnil, T., et al., Myocardial adipose triglyceride lipase overexpression protects diabetic mice from the development of lipotoxic cardiomyopathy. Diabetes, 2013. 62(5): p. 1464-1477.

70. Banerjee, R., et al., Evidence of a Direct Effect of Myocardial Steatosis on LV Hypertrophy and Diastolic Dysfunction in Adult and Adolescent Obesity. JACC: Cardiovascular Imaging, 2015.

71. Stanley, W.C., et al., Pyruvate dehydrogenase activity and malonyl CoA levels in normal and ischemic swine myocardium: effects of dichloroacetate. Journal of molecular and cellular cardiology, 1996. 28(5): p. 905-914.

72. Wynn, R.M., et al., Pyruvate dehydrogenase kinase-4 structures reveal a metastable open conformation fostering robust core-free basal activity. Journal of Biological Chemistry, 2008. 283(37): p. 25305-25315.

73. Curry, S.H., et al., Plasma concentrations and metabolic effects of intravenous sodium dichloroacetate. Clinical Pharmacology \& Therapeutics, 1985. 37(1): p. 89-93. 
74. Evans, O.B. and P.W. Stacpoole, Prolonged hypolactatemia and increased total pyruvate dehydrogenase activity by dichloroacetate. Biochemical pharmacology, 1982. 31(7): p. 12951300.

75. Eichner, H.L., P.W. Stacpoole, and P.H. Forsham, Treatment of streptozotocin diabetes with di-isopropylammonium dichloroacetate (DIPA). Diabetes, 1974. 23(3): p. 179-182.

76. Stacpoole, P.W., G.W. Moore, and D.M. Kornhauser, Metabolic effects of dichloroacetate in patients with diabetes mellitus and hyperlipoproteinemia. New England Journal of Medicine, 1978. 298(10): p. 526-530.

77. Le Page, L.M., et al., Increasing Pyruvate Dehydrogenase Flux as a Treatment for Diabetic Cardiomyopathy: A Combined 13C Hyperpolarized Magnetic Resonance and Echocardiography Study. Diabetes, 2015: p. db141560.

78. Nicholl, T.A., G.D. Lopaschuk, and J.H. McNEILL, Effects of free fatty acids and dichloroacetate on isolated working diabetic rat heart. American Journal of Physiology-Heart and Circulatory Physiology, 1991. 261(4): p. H1053-H1059.

79. Bersin, R.M., et al., Improved hemodynamic function and mechanical efficiency in congestive heart failure with sodium dichloroacetate. Journal of the American College of Cardiology, 1994. 23(7): p. 1617-1624.

80. Kaufmann, P., et al., Dichloroacetate causes toxic neuropathy in MELAS A randomized, controlled clinical trial. Neurology, 2006. 66(3): p. 324-330.

81. Shroads, A.L., et al., Human polymorphisms in the glutathione transferase zeta 1/maleylacetoacetate isomerase gene influence the toxicokinetics of dichloroacetate. The Journal of Clinical Pharmacology, 2012. 52(6): p. 837-849.

82. Ferriero, R., et al., Phenylbutyrate therapy for pyruvate dehydrogenase complex deficiency and lactic acidosis. Science translational medicine, 2013. 5(175): p. 175ra31-175ra31.

83. Ferriero, R., et al., Differential inhibition of PDKs by phenylbutyrate and enhancement of pyruvate dehydrogenase complex activity by combination with dichloroacetate. Journal of inherited metabolic disease, 2015: p. 1-10. 
84. Tso, S.-C., et al., Structure-guided development of specific pyruvate dehydrogenase kinase inhibitors targeting the ATP-binding pocket. Journal of Biological Chemistry, 2014. 289(7): p. $4432-4443$.

85. Kantor, P.F., et al., The antianginal drug trimetazidine shifts cardiac energy metabolism from fatty acid oxidation to glucose oxidation by inhibiting mitochondrial long-chain 3-ketoacyl coenzyme A thiolase. Circulation Research, 2000. 86(5): p. 580-588.

86. Allibardi, S., et al., Effects of trimetazidine on metabolic and functional recovery of postischemic rat hearts. Cardiovascular drugs and therapy, 1998. 12(6): p. 543-549.

87. Fragasso, G., et al., Effects of metabolic modulation by trimetazidine on left ventricular function and phosphocreatine/adenosine triphosphate ratio in patients with heart failure. European heart journal, 2006. 27(8): p. 942-948.

88. Ussher, J.R., et al., Trimetazidine Therapy Prevents Obesity-Induced Cardiomyopathy in Mice. Canadian Journal of Cardiology, 2014. 30(8): p. 940-944.

89. Ussher, J.R., et al., Treatment with the 3-Ketoacyl-CoA Thiolase Inhibitor Trimetazidine Does Not Exacerbate Whole-Body Insulin Resistance in Obese Mice. Journal of Pharmacology and Experimental Therapeutics, 2014. 349(3): p. 487-496.

90. Kuzmicic, J., et al., Trimetazidine prevents palmitate-induced mitochondrial fission and dysfunction in cultured cardiomyocytes. Biochemical pharmacology, 2014. 91(3): p. 323-336.

91. Clarke, B., K.M. Wyatt, and J.G. McCormack, Ranolazine increases active pyruvate dehydrogenase in perfused normoxic rat hearts: evidence for an indirect mechanism. Journal of molecular and cellular cardiology, 1996. 28(2): p. 341-350.

92. Maier, L., et al., Ranolazine for the treatment of diastolic heart failure in patients with preserved ejection fraction: results from the RALI-DHF study. Journal of the American College of Cardiology, 2012. 59(13s1): p. E865-E865.

93. Drucker, D.J., The biology of incretin hormones. Cell metabolism, 2006. 3(3): p. 153-165.

94. Zhao, T., et al., Direct effects of glucagon-like peptide-1 on myocardial contractility and glucose uptake in normal and postischemic isolated rat hearts. Journal of Pharmacology and Experimental Therapeutics, 2006. 317(3): p. 1106-1113. 
95. Nikolaidis, L.A., et al., Recombinant glucagon-like peptide-1 increases myocardial glucose uptake and improves left ventricular performance in conscious dogs with pacing-induced dilated cardiomyopathy. Circulation, 2004. 110(8): p. 955-961.

96. Lewis, A.J., et al., Hyperpolarized $13 C$ and $31 P$ magnetic resonance spectroscopy identify pyruvate dehydrogenase as a therapeutic target in obesity cardiomyopathy. Journal of Cardiovascular Magnetic Resonance, 2015. 17(Suppl 1): p. O16.

97. Aroor, A.R., et al., Dipeptidylpeptidase inhibition is associated with improvement in blood pressure and diastolic function in insulin-resistant male Zucker obese rats. Endocrinology, 2013. 154(7): p. 2501-2513.

98. Bostick, B., et al., Dipeptidyl peptidase inhibition prevents diastolic dysfunction and reduces myocardial fibrosis in a mouse model of Western diet induced obesity. Metabolism, 2014. 63(8): p. 1000-1011.

99. Noyan-Ashraf, M.H., et al., A glucagon-like peptide-1 analog reverses the molecular pathology and cardiac dysfunction of a mouse model of obesity. Circulation, 2013. 127(1): p. $74-85$.

100. Flint, A., et al., Glucagon-like peptide 1 promotes satiety and suppresses energy intake in humans. Journal of Clinical Investigation, 1998. 101(3): p. 515. 


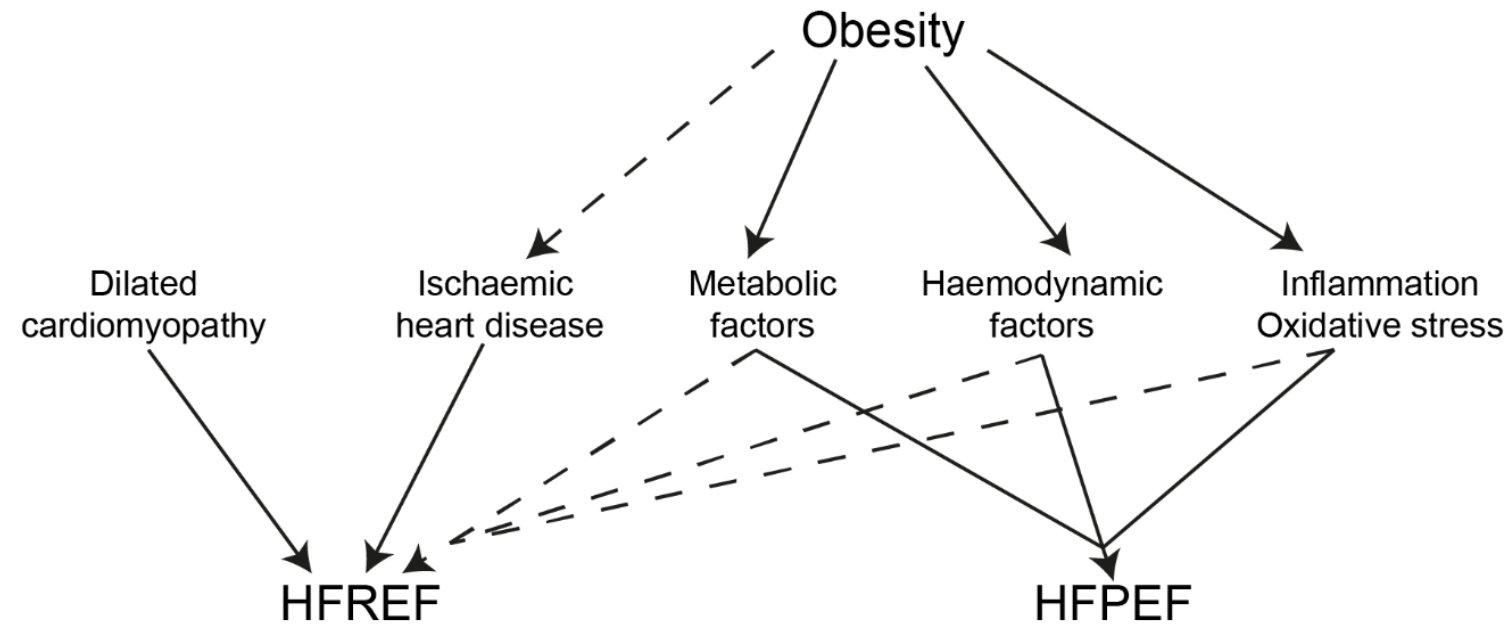

Figure 1: Obesity is independently associated with the development of heart failure (usually HFPEF) through a variety of mechanisms, but the same mechanisms are also likely to be detrimental to HFREF due to other causes such as dilated cardiomyopathy or ischaemic heart disease, for which obesity is a risk factor. 


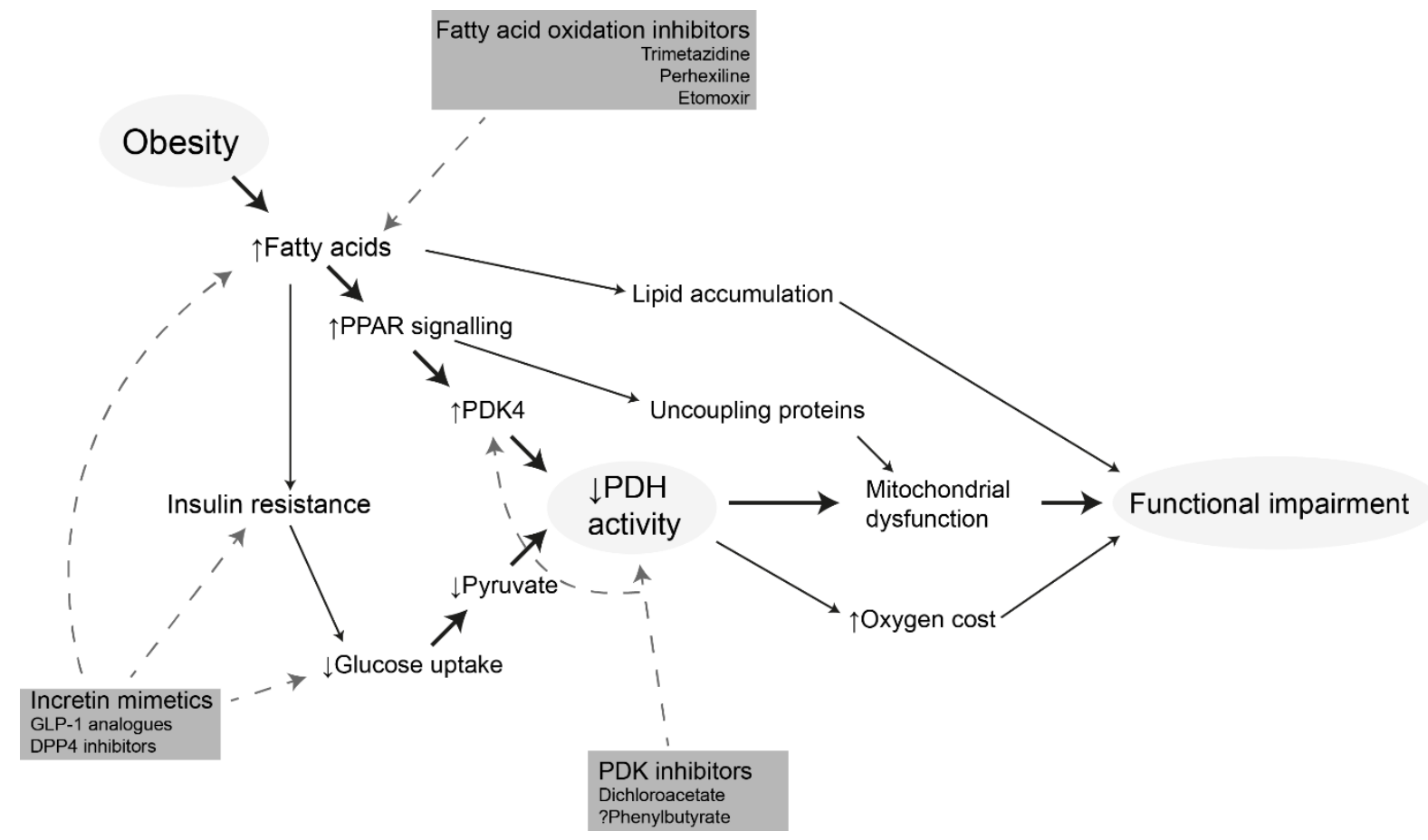

Figure 2: Overview of currently available pharmacological therapies which directly and indirectly increase PDH activity and improve cardiac function in obesity. 